\title{
Fundamentals of neurogastroenterology
}

\author{
J D Wood, D H Alpers, P L R Andrews
}

Co-Chair, Committee on Basic Science: Brain-Gut, Multinational Working Teams to Develop Diagnostic Criteria for Functional Gastrointestinal Disorders,

Departments of Physiology and Internal Medicine, The Ohio State University College of Medicine and Public Health, Columbus, Ohio, USA

J D Wood

Co-Chair, Committee on Basic Science: Brain-Gut, Gastroenterology Division, Department of Medicine,

Washington University School of Medicine, St Louis, Missouri, USA D H Alpers

Department of Physiology, St Georges Hospital Medical School, London, UK P L R Andrews

Correspondence to: Jackie D Wood, Ph.D, Professor of Physiology and Internal Medicine, Department of Physiology, College of Medicine, The Ohio State University, 302 Hamilton Hall, 1645 Neil Avenue, Columbus, Ohio 43210, USA. Email: wood.13@osu.edu

\begin{abstract}
Current concepts and basic principles of neurogastroenterology in relation to functional gastrointestinal disorders are reviewed. Neurogastroenterology is emphasized as a new and advancing subspecialty of clinical gastroenterology and digestive science. As such, it embraces the investigative sciences dealing with functions, malfunctions, and malformations in the brain and spinal cord, and the sympathetic, parasympathetic and enteric divisions of the autonomic innervation of the digestive tract. Somatomotor systems are included insofar as pharyngeal phases of swallowing and pelvic floor involvement in defecation, continence, and pelvic pain are concerned. Inclusion of basic physiology of smooth muscle, mucosal epithelium, and the enteric immune system in the neurogastroenterologic domain relates to requirements for compatibility with neural control mechanisms. Psychologic and psychiatric relations to functional gastrointestinal disorders are included because they are significant components of neurogastroenterology, especially in relation to projections of discomfort and pain to the digestive tract.

(Gut 1999;45(Suppl II):II6-II16)
\end{abstract}

Keywords: enteric nervous system; brain-gut axis; autonomic nervous system; nausea; gut motility; mast cells; gastrointestinal pain; Rome II

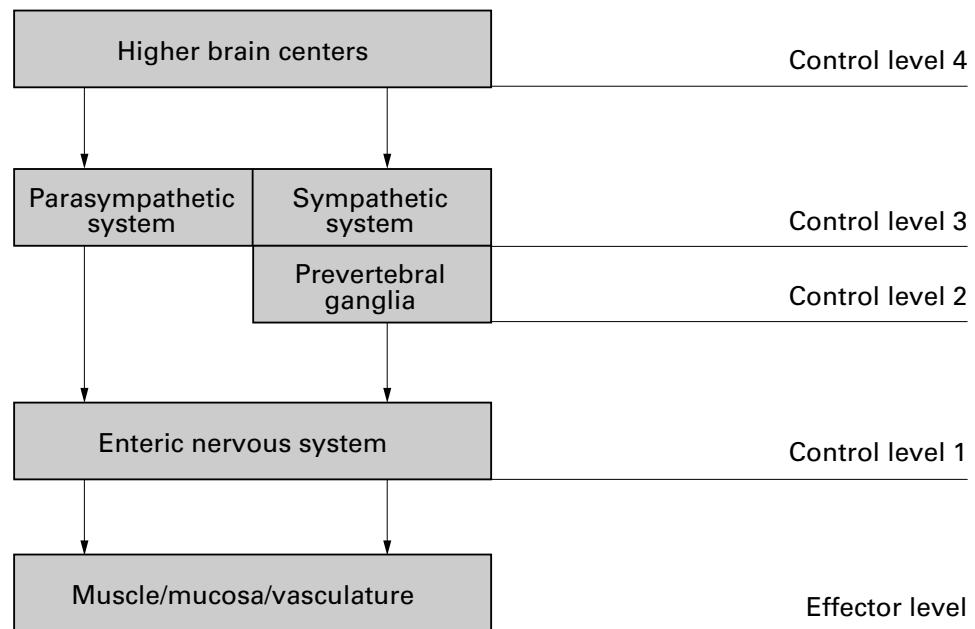

Figure 1 Neural control of the gut is hierarchic with four basic levels of integrative organization. Level 1 is the enteric nervous system (ENS) which behaves like a local minibrain. The second level of integrative organization is in the prevertebral sympathetic ganglia. The third and fourth levels are within the central nervous system (CNS). Sympathetic and parasympathetic signals to the digestive tract originate at level 3 and represent the final common pathways for outflow of information from the CNS to the gut. The fourth level includes higher brain centers that provide input for integrative functions at level 3.
Enteric innervation

Neural networks for control of digestive functions are positioned in the brain, spinal cord, prevertebral sympathetic ganglia, and in the walls of the specialized organs that make up the digestive system. Control involves an integrated hierarchy of neural centers. Starting at the level of the gut, fig 1 illustrates four levels of integrative organization. Level 1 is the enteric nervous system (ENS), which has local circuitry for integrative functions independent of extrinsic nervous connections. The second level of integration occurs in the prevertebral sympathetic ganglia where peripheral reflex pathways are influenced by preganglionic sympathetic fibers from the spinal cord. Levels 3 and 4 are within the central nervous system (CNS). At the third level, sympathetic and parasympathetic outflow to the gut is determined in part by reflexes with sensory fibers that travel with autonomic nerves. The fourth level includes higher brain centers that supply descending signals that are integrated with incoming sensory signals at level 3. The neural networks at level 1 within the walls of the gut integrate contraction of the muscle coats, transport across the mucosal lining and intramural blood flow into organized patterns of behavior. These networks form the ENS, which is considered to be one of the three subdivisions of the autonomic nervous system together with sympathetic and parasympathetic divisions. Nervous malformations and malfunctions in these systems are increasingly recognized as underlying factors in functional gastrointestinal disorders (FGID).

The musculature, mucosal epithelium, and vasculature are the gut's effector systems. Global behavior of the organ at any moment reflects neurally integrated activity of these systems. The nervous system coordinates activity of the primary effectors to produce meaningful patterns of behavior for the whole organ. The ENS is a local minibrain within which is stored a library of programs for different patterns of gut behavior. Digestive, interdigestive, and emetic patterns of intestinal behavior reflect outputs from three respective programs. For example, during emesis, propulsion in the upper small intestine is reversed for rapid movement of the contents toward the open pylorus and relaxed stomach. This program can be called up from the library either by

Abbreviations used in this paper: CNS, central nervous system; ENS, enteric nervous system; FGID, functional gastrointestinal disorder; NTS, nucleus tractus solitarius; DVN, dorsal motor nucleus of the vagus; IBS, irritable bowel syndrome; ICC, interstitial cells of Cajal; 5-HT, 5-hydroxytryptamine; TRH thyrotropin releasing hormone; CRF, corticotropin releasing factor. 


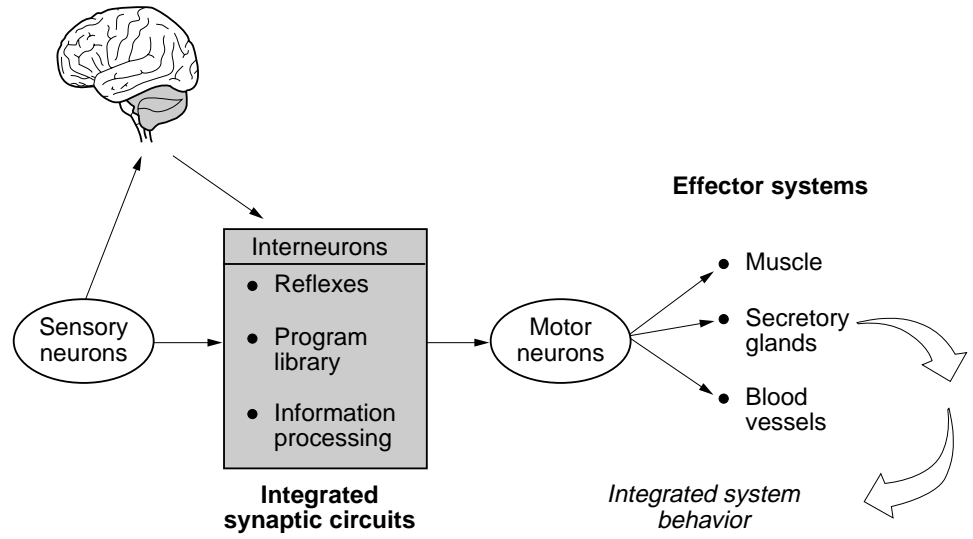

Figure 2 The conceptual model for the enteric nervous system (ENS) is the same as for the central nervous system (CNS). Sensory neurons, interneurons, and motor neurons are connected synaptically for flow of information from sensory neurons to interneuronal integrative networks to motor neurons to effector systems. The ENS organizes and coordinates the activity of each effector system into meaningful behavior of the integrated organ. Bi-directional communication occurs between the CNS and ENS.

commands from the brain or by local sensory detection of noxious substances in the lumen.

Structure, function, and neurochemistry of enteric ganglia differ significantly from other autonomic ganglia. Unlike other autonomic ganglia that function mainly as relay distribution centers for signals transmitted from the CNS, ENS ganglia are interconnected to form a nervous system with mechanisms for integration and processing of information like those found in the brain and spinal cord. On this basis, the ENS is sometimes referred to as the brain-in-the-gut or enteric minibrain.

Many properties of the ENS resemble the $\mathrm{CNS}^{12}$ and the conceptual model is the same (fig 2). Like the CNS, the ENS works with three functional categories of neurons identified as sensory, inter-, and motor neurons.

Sensory neurons have receptor regions specialized for detecting changes in thermal, chemical, or mechanical stimulus energy. The receptor regions transform changes in stimulus energy into signals coded by action potentials that subsequently are transmitted along sensory nerve fibers to other points in the nervous system.

Interneurons are connected by synapses into networks that process sensory information and control the behavior of motor neurons. Multiple connections among many interneurons form "logic" circuits that decipher action potential codes from sensory neurons and signals from elsewhere in the nervous system. These are recognized as integrative or reflex circuits because they organize reflex responses to sensory inputs.

Motor neurons are the final common pathways for transmission of control signals to the effector systems. In the digestive tract, motor signals may initiate, sustain, or suppress the behavior of the effector depending on the kind of transmitter released.

\section{Reflexes and pattern generators}

Reflexes are a form of neurally-mediated behavior of effector systems that occurs in response to stimulation of sensory neurons. Reflex behavior is stereotypical. For example, the response in the wall of the intestine to distension or mucosal stroking is a reflex contraction of the circular muscle coat above the site of stimulation and inhibition of the circular muscle below the site. This pattern of behavior is reproduced each time mechanoreceptors are activated by stretch of the wall or deformation of the mucosa. This behavioral pattern, like that of all reflexes, mirrors the output of a set of fixed "hardwired" connections within the interneuronal circuitry.

Pattern generators are neural networks that generate rhythmic or repetitive behavior in effector systems of animal phyla ranging from invertebrates to higher vertebrates including humans. They are formed by interneuronal synaptic connections that are preprogramed to produce an adaptive pattern of effector behavior. Pattern-generating circuitry consists of motor programs that signal motor neurons for control of repetitive cyclical behaviors. The sequence of events in stereotyped repetitions of motor outflow to the effector system is determined by the program circuit. Programmed motor behavior, unlike reflex behavior, does not require sensory input to start the program, and feedback information from sensory neurons is unnecessary for sequencing of the steps in the program. For many of the behaviors generated by programmed motor circuits (e.g., chewing, swallowing, breathing), the entire sequence of the motor program may be initiated by input signals from a single neuron called a command neuron. Cyclic patterns of secretory and contractile behavior seen in the large intestine in response to histamine release from enteric mast cells is an example of the output of pattern generating circuitry in the ENS. $^{34}$

\section{Central command signals}

The vagus nerves have long been recognized as the major transmission pathway for control signals from the brain to the digestive tract, whereas the general neurophysiological mechanisms underlying the effects of vagal nerve stimulation on the upper gut have been clarified only recently. New awareness of the independent integrative properties of the ENS has led to revision of earlier concepts of mechanisms of vagal influence. Earlier concepts of vagal innervation presumed that ganglia of the digestive tract were the same as parasympathetic ganglia in other visceral systems where the ganglia generally have a relay distribution function. These previous concepts supposed that parasympathetic innervation of the gut was similar. Efferent vagal fibers were believed to form synapses directly with ganglion cells that innervated the cells of the effector systems. This concept, illustrated in fig 3, is inconsistent with current evidence and should be abandoned.

The earlier concept placed the "computer" entirely within the brain, whereas, current concepts place "microprocessor" circuits within the wall of the gut in close proximity to the effector systems. Numbers of neurons equal to those of the spinal cord are present in the ENS (i.e., $\sim 1 \times 10^{8}$ ). This large amount, which 


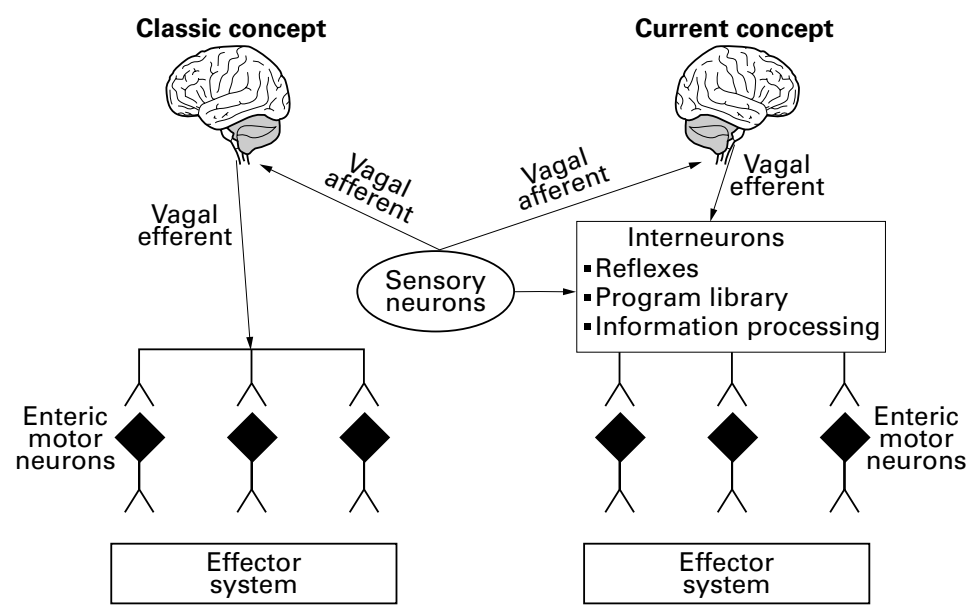

Figure 3 Classic outmoded and current concepts of relations between the brain and the digestive tract. The classic concept viewed parasympathetic efferents (e.g., vagal efferents) as synapsing directly with enteric motor neurons, as illustrated on the left side of the diagram. In the current concept, parasympathetic efferent fibers transmit command signals from the brain to the integrative and motor program circuitry of the enteric nervous system minibrain as shown on the right side of the diagram.

evidently is required for program control of the digestive processes, would greatly expand the volume of the CNS if situated there. Rather than having the neural control circuits packed exclusively within the CNS and transmitting every byte of control information over long transmission lines, vertebrate animals have most of the circuits for automatic feedback control located in close proximity to the effector systems.

Figure 3 illustrates the current concept of central involvement in gut function. Local integrative circuits of the ENS are organized for program operations independent of input from the CNS. Subsets of neural circuits are preprogramed for control of distinct patterns of behavior in each effector system and for the coordination of activity of multiple systems. Enteric motor neurons are the final common transmission pathways for the variety of different programs and reflex circuits required for ordered gut function.

Rather than controlling individual motor neurons, messages transmitted by parasympathetic efferent fibers are command signals for the activation of expanded blocks of integrated circuits positioned in the gut wall. This explains the strong influence of a small number of vagal efferent fibers (approximately 10\% of vagal fibers are efferent) on motility and other effector systems over extended regions of the stomach or intestine. In this respect, the ENS is analogous to a microcomputer with its own independent software, whereas the brain is like a larger mainframe with extended memory and processing circuits that receive information from and issue commands to the enteric computer.

\section{Higher brain centers}

Final common pathways for output from higher centers to the gut exit the brain in efferent vagal fibers and descending pathways in the spinal cord that connect to sympathetic preganglionic neurons in the thoraco-lumbar region and parasympathetic preganglionic neu- rons in the sacral region. Several higher brain regions transmit to these outflow centers. Frontal regions of the cerebral cortex, bed nucleus of the stria terminalis, paraventricular nucleus of the hypothalamus, and the central nucleus of the amygdala project to the vagal outflow center in the medulla oblongata. These areas share information with the limbic regions where emotional responses to sensory input from the outside world as well as signals of volitional origin are processed.

Vagal afferent fibers from the upper gastrointestinal tract form synapses in the nucleus tractus solitarius (NTS). They are thought to mediate non-painful physiologic sensations (e.g., distension, satiety and nausea). Evidence for a role in the perception of noxious stimulation is equivocal. The NTS is the relay station for transfer of vagal afferent information from the gut to higher brain centers. Information relayed by the NTS influences the outflow of both volitional and non-volitional signals from higher brain centers.

Linked interactions between higher brain centers, emotional state and gastrointestinal disorders are well recognized. Abdominal pain, diarrhea, nausea, altered food intake, and emesis can all be manifestations of emotional or traumatic stress. The same symptoms following stress can occur in psychiatrically normal individuals, as well as in those with psychiatric illness. Findings that antidepressant medications relieve some FGID symptoms is evidence of disorder in the higher brain centers that influence the outflow of commands to the gut. Nevertheless, the effect is not evident for all antidepressants and is not necessarily related to effects on mood. ${ }^{5}$ The stress-related symptoms and behavioral changes (i.e., sleep disturbances, muscle tension, pain, altered diet, abnormal illness behavior) associated with FGIDs probably reflect subtle malfunctions in the brain circuits responsible for interactions of higher cognitive functions and central centers that determine outputs to the gastrointestinal tract, and not to psychiatric illness alone.

\section{Vago-vagal reflexes}

Vagal integrative centers in the brain are more directly involved in the control of the specialized digestive functions of the esophagus, stomach and the functional cluster of duodenum, gall bladder, and pancreas than in the distal small bowel and large intestine. The circuits in the dorsal vagal complex and their interactions with higher centers are responsible for the rapid and more precise control required for adjustments to rapidly changing conditions in the upper digestive tract during anticipation, ingestion, and digestion of meals of varied composition.

A reflex circuit known as the vago-vagal reflex underlies moment-to-moment adjustments required for optimal digestive function in the upper digestive tract. The sensory side of the reflex arc consists of vagal afferent neurons connected with a variety of sensory receptors specialized for detection and signaling of mechanical parameters such as muscle tension and mucosal brushing, or luminal chemical 
parameters such as $\mathrm{pH}$, osmolarity and glucose concentration. The sensory neurons are synaptically connected with neurons in the dorsal motor nucleus of the vagus (DVN) and in the NTS. The NTS, which lies directly above the DVN, makes synaptic connections with the neuronal pool in the DVN. A synaptic neuropile formed by processes from neurons in both nuclei tightly links the two into an integrative center, which together with the area postrema and nucleus ambiguus, form the dorsal vagal complex. The dorsal vagal neurons are second or third order neurons representing the efferent arm of the reflex circuit. They are the final common pathways out of the brain to the enteric circuits responsible for control and coordination of the behavior of the muscular, secretory, and circulatory systems of the gut.

Efferent vagal fibers form synapses with neurons in the ENS to activate circuits which ultimately drive outflow of signals in motor neurons to the effector systems. When the effector system is the musculature, its innervation consists of both inhibitory and excitatory motor neurons that participate in reciprocal control. If the effector systems are gastric glands or digestive glands of the duodenal cluster unit, the secretomotor neurons are excitatory and stimulate secretory behavior.

The circuits for central nervous control of the upper gastrointestinal tract are organized much like those dedicated to control of skeletal muscle movements where fundamental reflex circuits are located in the spinal cord. Inputs to the spinal reflex circuits (e.g., monosynaptic reflexes) from higher order integrative centers in the brain (e.g., motor cortex and basal ganglia) complete the neural organization of skeletal muscle motor control. Memory, processing of incoming information from outside the body and integration of proprioceptive information are ongoing functions of higher brain centers responsible for intelligent organization of the outflow to the skeletal muscles emanating from the basic spinal reflex circuits. The basic connections of the vago-vagal reflex circuit are like somatic motor reflexes in being "fine tuned" by higher brain centers.

The dorsal vagal complex has extensive connections for information-sharing with both forebrain and brainstem centers. Sensory information into the NTS and area postrema is relayed to several rostral centers. The same rostral centers reciprocate by projecting higher order information in descending connections to the vago-vagal reflex circuits. These interactions account for the effects of emotional state and external stimuli from the environment on functions of the digestive tract.

Synaptic transmission in the microcircuits of the dorsal vagal complex involves more than 30 neurotransmitters. These include acetylcholine, biogenic amines, amino acids, nitric oxide, and peptides, most of which are identified as neurotransmitters elsewhere in the brain and in the ENS.

\section{Sensory physiology}

The gut has mechano-, chemo-, and thermoreceptors. Cell bodies of these neurons are in nodose ganglia, dorsal root ganglia, and in the ENS. Mechanoreceptors sense mechanical events in the mucosa, musculature, serosal surface, and mesentery. They supply both the enteric minibrain and the CNS with information on stretch-related tension and muscle length in the wall and on the movement of luminal contents as they brush the mucosal surface. Mesenteric receptors code for gross movements of the organ. Chemoreceptors generate information on the concentration of nutrients, osmolarity and $\mathrm{pH}$ in the luminal contents. Thermoreceptors supply the brain with deep-body temperature data used in regulation and perhaps sensations of temperature change in the lumen. Presence in the gastrointestinal tract of nociceptors ("pain receptors"), equivalent to those connected with C-fibers and A-delta fibers elsewhere in the body, is likely but not unequivocally confirmed. ${ }^{6}$

Sensory information on the mechanical state of the musculature and distension of the visceral wall is coded by mechanoreceptors. Whether the neuronal cell bodies of intramuscular and mucosal mechanoreceptors belong to dorsal root ganglia, enteric ganglia, or both, is uncertain. ${ }^{2} 7$ Stretch sensitive mechanoreceptors have pathophysiologic importance because a consistent finding in patients diagnosed with the irritable bowel syndrome (IBS) is abnormally high sensitivity to stretch that translates into pain. ${ }^{89}$ The heightened sensitivity to distension and conscious awareness of the gastrointestinal tract experienced by patients with IBS is a generalized phenomenon throughout the gut including the esophagus. ${ }^{10}$ The mechanism is unclear. However, three general explanations are apparent: (1) exaggerated signals from sensitized mechanoreceptors may be accurately decoded by the brain as hyperdistension; (2) malfunctioning brain circuits may be misinterpreting accurate information; (3) combined sensing and central processing malfunction could be involved.

Hyposensory perception, particularly in the rectosigmoid region, is at the opposite extreme of gastrointestinal sensory abnormality. Sensory suppression in this region of the gut, either in the pathway for recto-anal stretch reflexes or in the transmission pathway from the rectosigmoid to conscious perception of distension, can be an underlying factor in the pathogenesis of chronic constipation and associated symptoms. ${ }^{11}$

Conscious sensations arising from mechanical stimulation in the specialized compartments of the digestive tract in humans include pressure, fullness, nausea, and pain. Chemical stimuli (e.g., glucose, fatty acids, and amino acids) evoke discharge in gastrointestinal afferent fibers; nevertheless, it is unlikely that this normally gives rise to conscious sensation other than perhaps hunger and satiety. Sensations of pain are transmitted mainly by dorsal root afferents that accompany the splanchnic nerves. Electrical stimulation of splanchnic nerves in humans evokes severe pain that is not relieved by vagotomy. ${ }^{12}$ Splanchnic afferents seem to be involved in the sensation of nausea 
because nausea can be evoked by gastric distension in patients with bilateral vagotomy. ${ }^{13}$ This suggests that stimulation of splanchnic afferents can evoke the sensation, but does not exclude vagal involvement. Vagal stimulation has long been implicated as a factor in nausea. ${ }^{14}$ In contrast to vagal afferents, stimulation of greater splanchnic nerves does not evoke an emetic response in animal models.

Low-threshold afferents respond to innocuous levels of distension and contraction; high-threshold afferents respond only when distension is greater than a set threshold. Lowthreshold mechanoreceptors are presumed to be the sensory component of normal autonomic regulatory reflexes (e.g., vago-vagal reflexes). It is unknown for certain whether activity in low-threshold pathways reaches the level of conscious perception; nevertheless, it is likely that some non-painful sensations such as fullness, the presence of gas, or perhaps nausea are derived from this kind of activity. Highthreshold afferents are thought to be the sensory analogs of sharp-localized pain in organs such as the gall bladder where pain is the only consciously perceived sensation. ${ }^{15}$ Cervero and Jänig ${ }^{6}$ suggested that distension can evoke sensations ranging from mild fullness to intense pain and that activation of differing proportions of low- and highthreshold mechanoreceptors could account for the range of sensations. Acute visceral pain may emerge from activation of high-threshold nociceptive fibers; whereas, chronic forms of visceral pain could be attributed to sensitization of both types of mechanoreceptors by conditions such as inflammation or ischemia. Application of irritants to the large intestinal mucosa in animals lowers the threshold and sensitizes both the high- and low-threshold distension sensitive afferents. Another class of splanchnic afferents termed silent nociceptors is also suspected in chronic pain.

Silent nociceptors are sensory afferents that normally do not respond to the strongest of distending stimuli. This group of normally silent receptors appears to become sensitized by inflammatory mediators. Spontaneous action potential discharge and responses to normally innocuous mechanical distension occur after sensitization.

\section{Enteric motor physiology}

The motor neuron pool of the ENS consists of excitatory and inhibitory neurons (fig 2). Excitatory motor neurons release neurotransmitters that evoke muscle contractions and mucosal secretion. Acetylcholine and substance $\mathrm{P}$ are the main neurotransmitters released from excitatory motor neurons to evoke contraction of the muscles. ${ }^{16}$ Acetylcholine and vasoactive intestinal peptide are excitatory neurotransmitters that evoke secretion from intestinal crypts. ${ }^{17}$

Inhibitory motor neurons release neurotransmitters that suppress contractile activity of the musculature. Adenosine triphosphate, vasoactive intestinal peptide, pituitary adenylate cyclase activating peptide, and nitric oxide are implicated as inhibitory neurotransmitters at neuromuscular junctions in the gut. $^{18} 19$

The functional significance of inhibitory motor neurons is related to the specialized physiology of the musculature. ${ }^{2}$ The intestinal musculature behaves as a self-excitable electrical syncytium consisting of interstitial cells of Cajal (ICCs) that function as pacemakers integrated with the bulk musculature, which generates forces for propulsion. This implies that action potentials and pacemaker potentials spread from muscle fiber to muscle fiber in three dimensions. The action potentials trigger phasic contractions as they spread through the musculature. The ICCs are a non-neural pacemaker system of electrical slow waves that account for the self-excitable characteristics of the integrated system. In this construct, the electrical slow waves are an extrinsic factor to which the circular muscle responds.

Consideration of the functional characteristics of the musculature raises the question of why the circular muscle fails to respond with action potentials and contractions to all pacemaker cycles and why action potentials and contractions do not spread in the syncytium throughout the entire length of intestine each time they occur? Answers to the these questions lie in the functional significance of enteric inhibitory motor neurons.

The circular muscle can only respond to a myogenic pacemaker (electrical slow wave) when the inhibitory motor neurons in a segment of intestine are switched off by input from other neurons in the control circuits. Likewise, action potentials and associated contractions can propagate only into disinhibited regions of the musculature. This means that activity states of inhibitory neurons determine when the omnipresent slow waves initiate a contraction, as well as the distance and direction of propagation once the contraction has begun.

Inhibitory motor neurons to the circular muscle discharge continuously and action potentials and contractions in the muscle occur only when the inhibitory neurons are switched off by input from interneurons in the control circuits. In sphincters, the inhibitory neurons are normally quiescent and are switched on with timing appropriate for coordination of the opening of the sphincter with physiological events in adjacent regions. When this occurs, the inhibitory neurotransmitter relaxes ongoing muscle contraction in the sphincteric muscle and prevents excitation-contraction in the adjacent muscle from spreading into and closing the sphincter. In non-sphincteric circular muscle, the state of activity of inhibitory motor neurons determines the length of a contracting segment by controlling the distance of spread of action potentials within the threedimensional electrical geometry of the syncytium. Contraction can occur in segments in which ongoing inhibition has been switched off, while adjacent segments with continuing inhibitory activity cannot contract. The boundaries of the contracted segment reflect the transition zone from inactive to active 
inhibitory motor neurons. The directional sequence in which the inhibitory motor neurons are switched off establishes the direction of propagation of the contraction. Normally, they are switched off in the aboral direction, resulting in contractile activity that propagates in the aboral direction. During vomiting, the inhibitory motor neurons must be switched off in the reverse sequence to account for small intestinal propulsion that travels toward the stomach.

In general, any treatment or condition that ablates the intrinsic inhibitory neurons results in tonic contracture and "achalasia" of the intestinal circular muscle. Several circumstances that involve functional ablation of the intrinsic inhibitory neurons are associated with conversion from a hypocontractile condition of the circular muscle to a hypercontractile state. All evidence suggests that some of the intrinsic inhibitory neurons are tonically active, and that blockade or ablation of these neurons releases the circular muscle from the inhibitory influence. ${ }^{2}$ The behavior of the muscle in these cases is tonic contracture and disorganized phasic contractile activity reminiscent of fibrillation.

\section{Disinhibitory motor disease}

The physiology of neuromuscular relations in the intestine predicts that spasticity and "achalasia" will accompany any condition where inhibitory motor neurons are destroyed. Without inhibitory control, the self-excitable smooth muscle contracts continuously and behaves as an obstruction. This happens because the muscle is freed to respond to the pacemaker (electrical slow waves) with contractions that propagate without amplitude, distance, or directional control. Contractions spreading in the uncontrolled syncytium collide randomly resulting in fibrillation-like behavior in the affected intestinal segment.

Loss or malfunction of inhibitory motor neurons is the pathophysiologic basis of disinhibitory motor disease. It underlies several forms of chronic intestinal pseudo-obstruction and sphincteric achalasia. Neuropathic degeneration is a progressive disease that in its earlier stages may be manifest as symptoms confused with FGID.

Functional gastrointestinal disorders and chronic intestinal pseudo-obstruction

The neuropathic form of chronic intestinal pseudo-obstruction is linked with neuropathic degeneration in the ENS. Failure of propulsive motility in the affected length of neuropathic bowel reflects loss of the neural microcircuits that program and control the repertoire of motility patterns required for the necessary functions of that region of bowel. Pseudoobstruction occurs in part because contractile behavior of the circular muscle is hyperactive but disorganized in the denervated regions. ${ }^{20}$ Manometrically determined hyperactivity is a diagnostic sign of the neuropathic form of chronic small bowel pseudo-obstruction. The hyperactive and disorganized contractile behavior reflects the absence of inhibitory nervous control of the muscles that are self-excitable (autogenic) when released from the braking action imposed by inhibitory motor neurons. Chronic pseudo-obstruction in these cases appears to be symptomatic of advanced stages of a progressive enteric neuropathy. Retrospective review of patients' records suggests that FGID symptoms can be an expression of early stages of the neuropathy.

Degenerative non-inflammatory and inflammatory enteric neuropathies are two forms of the disease that culminate in pseudoobstruction. Non-inflammatory neuropathies can be either familial or sporadic. ${ }^{21}$ In the autosomal recessive form, the neuropathologic findings include a notable reduction in the number of neurons in both myenteric and submucous plexuses, and the presence of round, eosinophilic intranuclear inclusions in about $30 \%$ of the residual neurons. Histochemical and ultrastructural analysis revealed that the inclusions are not viral particles, but rather proteinaceous material forming filaments. ${ }^{22}{ }^{23}$

Degenerative inflammatory enteric neuropathies are characterized by a dense inflammatory infiltrate confined to enteric ganglia. Paraneoplastic syndrome, Chagas disease and idiopathic degenerative disease are recognizable forms of pseudo-obstruction related to inflammatory neuropathies.

Idiopathic inflammatory degenerative neuropathy occurs unrelated to neoplasms, infectious conditions or other known diseases. ${ }^{24-26}$ DeGiorgio and colleagues ${ }^{25}$ and Smith and colleagues $^{26}$ described two small groups of patients with early complaints of symptoms similar to FGID, which progressively worsened, and were later diagnosed as idiopathic degenerative inflammatory neuropathy based on full-thickness biopsy samples taken during exploratory laparotomy that revealed chronic intestinal pseudo-obstruction. Each patient had inflammatory infiltrates localized to the myenteric plexus. Serum samples from the two cases reported by Smith et al had circulating antibodies against enteric neurons similar to those found in secondary inflammatory neuropathies (i.e., anti-Hu), but with different immunolabeling patterns characterized by prominent cytoplasmic rather than nuclear staining. ${ }^{26}$

Recognition of the complex functions of the enteric minibrain prompts the conclusion that early neuropathic changes are expected to be manifest as functional symptoms that worsen with progressive neuronal loss. In diagnostic motility studies (e.g., manometry) degenerative loss of enteric neurons is reflected by hypermotility and spasticity ${ }^{20}$ because inhibitory motor neurons are included in the missing neuronal population.

\section{Nausea and vomiting}

Nausea and vomiting are common symptoms of FGIDs. Both are viewed as components of a neuroprotective mechanism against accidentally ingested toxins. ${ }^{27}$ Nausea is responsible for the genesis of an aversive response either by taste, sight, or smell such that the animal avoids the toxin on future occasions. Vomiting expels 
the toxin from the upper gastrointestinal tract, much like diarrhea and power propulsion accomplish a similar function in the lower gut. Nausea induces an aversive response linking the sensation to recently ingested food that contained the toxin. In humans, nausea can be more aversive than pain. Effects of inappropriate induction of nausea in the clinic are seen in patients undergoing anti-cancer chemotherapy who may experience reduced food intake, anticipatory emetic responses, and aversion to further courses of therapy. Aside from its adaptive advantage in evolution, some animals, including humans, experience nausea and vomiting as a symptom in response to an extended range of drugs, therapies, disease processes, and altered mental states.

The somatic motor acts of retching and vomiting are preceded by changes mediated by the autonomic nervous system including salivation, tachycardia, cutaneous vasoconstriction, sweating, relaxation of the proximal stomach, and retrograde propulsive contractions in the upper small bowel. Vomiting center is a shorthand term for the brainstem structures that contain the neural program for organization of both the autonomic and somatic motor outflow components in generation of the emetic response. Input to the vomiting center from vagal afferents, the area postrema, vestibular system, and higher brain structures can induce nausea and trigger the emetic pattern generator in humans. Input from abdominal vagal afferents is also a trigger for the vomiting center.

Several lines of evidence suggest that paracrine signaling from mucosal enteroendocrine cells to vagal afferent terminals, with major involvement of 5-hydroxytryptamine (5-HT) as the mediator, underlies signal transduction at the afferent terminal. This is assumed to underlie the efficacy of $5-\mathrm{HT}_{3}$ antagonists as antiemetic drugs. The vomiting center itself should be an ideal target for antiemetic drug therapy because a drug acting there could potentially block retching and vomiting irrespective of the initial trigger. Animal studies have identified several classes of agents that may work in this way. These include $5-\mathrm{HT}_{1 \mathrm{~A}}$ receptor agonists, ${ }^{28}$ opiate receptor agonists, ${ }^{29}$ the capsaicin analog resiniferatoxin, ${ }^{30}$ and neurokinin NK-1 receptor antagonists. ${ }^{31}$ Neurokinin antagonists block retching and vomiting induced by activation of vagal afferents with electrical stimulation, intragastric irritants, cisplatin, and radiation. They also block retching and vomiting evoked by stimulation of the area postrema with apomorphine or loperamide and stimulation of the vestibular system with motion. Early results from clinical trials suggest that the neurokinin antagonists block the sensation of nausea as well as retching and vomiting. This suggests action at a site before divergence of the pathways responsible for the sensation of nausea and the motor behavior of emesis. The most likely site of action is the NTS in the brain stem. ${ }^{31}$ Observations that a non-peptide neurokinin-1 receptor antagonist can block the emetic response to abdominal vagal afferent stimulation raises the possibility that this class of drugs may have potential for modifying other non-painful sensations arising from the upper digestive tract.

\section{Neuroimmunophysiologic paradigm for} functional gastrointestinal disorders

The enteric immune system is colonized by populations of immune/inflammatory cells that are constantly changing in response to luminal conditions and during pathophysiologic states. In its position in the colon, the mucosal immune system encounters one of the most contaminated of bodily interfaces with the outside world. The system is exposed daily to dietary antigens, bacteria, viruses, and toxins. Physical and chemical barriers at the epithelial interface do not exclude the large antigenic load in its entirety, causing the mucosal immune system to be chronically challenged.

Motor and secretory responses in the gut of animals sensitized to specific antigens (e.g., parasites, food antigens, bacterial toxins) suggest direct communication between the immune system and the ENS that may be normal or become pathologic. The communication results in adaptive behavior of the bowel in response to circumstances within the lumen that are threatening to the functional integrity of the whole animal. Communication is paracrine in nature and incorporates specialized sensing functions for specific antigens together with the capacity of the ENS for intelligent interpretation of the signals. Flow of information in immuno-neural integration starts with immune detection and signal transfer to the ENS. The enteric minibrain interprets the signal and responds by calling up from its program library a specific program of coordinated mucosal secretion and propulsive motility that functions to clear the antigenic threat from the intestinal lumen. Side effects of the program are symptoms of abdominal pain and diarrhea (fig 4).

The enteric immune system becomes sensitized by foreign antigens in the form of foodstuffs, toxins and invading organisms. Once the system is sensitized, a second exposure to the same antigen triggers predictable integrated behavior of the intestinal effector systems. ${ }^{32}$ Neurally coordinated activity of the musculature, secretory epithelium and blood vasculature results in organized behavior of the whole intestine that rapidly expels the antigenic threat. Recognition of an antigen by the sensitized immuno-neuro-apparatus leads to activation of a specialized propulsive motor program that is integrated with copious secretion of water, electrolytes, and mucus into the intestinal lumen. Detection by the enteric immune system and signal transmission to the enteric minibrain initiates the defensive behavior which is analogous to emetic defense in the upper gastrointestinal tract. The neurally organized pattern of muscle behavior that occurs in response to an offending antigen in the sensitized intestine is called power propulsion. This specialized form of propulsive motility forcefully and rapidly propels any material in the lumen over long distances and effectively 


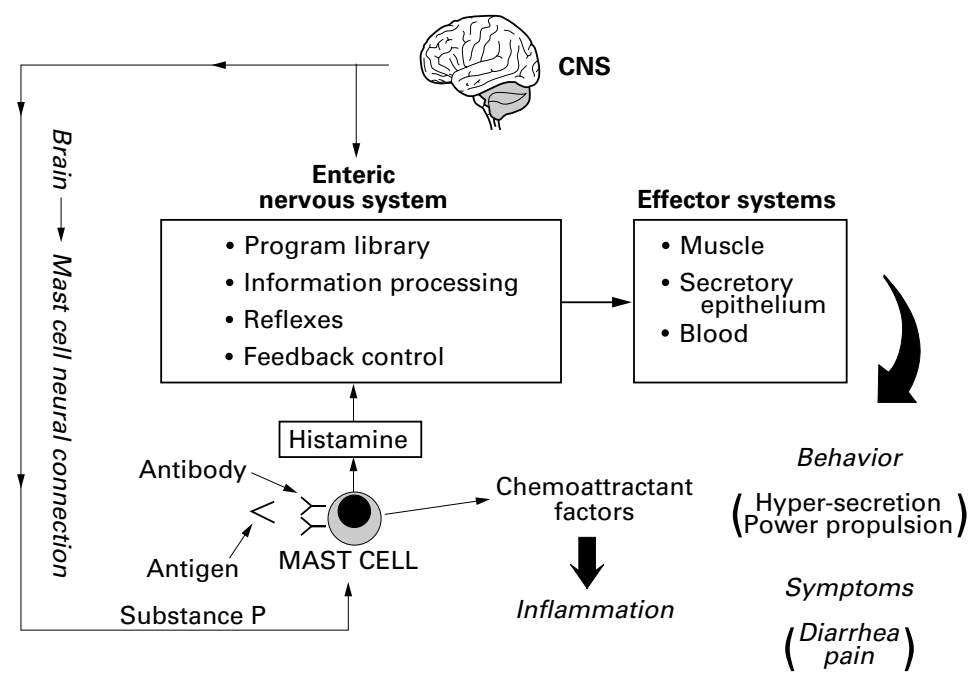

Figure 4 Conceptual model for enteric neuro-immunophysiology. The enteric nervous system (ENS) is a minibrain located in close apposition to the gastrointestinal effectors it controls. Enteric mast cells are in position to detect foreign antigens and signal their presence to the ENS. Stimulated mast cells release several paracrine mediators simultaneously. Some of the mediators signal the ENS whereas others act as attractant factors for

polymorphonuclear leucocytes responsible for acute inflammatory responses. The ENS responds to the mast cell signal by initiating a program of coordinated secretion and propulsive motility that expels the source of antigenic stimulation from the bowel. Symptoms of abdominal pain and diarrhea result from operation of the neural program. Neural inputs to mast cells from the brain stimulate simultaneous release of chemoattractant factors for inflammatory cells and chemical signals to the ENS with symptomatic consequences that mimic antigenic stimulation. CNS, central nervous system.

empties the lumen. Its occurrence is accompanied by abdominal discomfort and diarrhea. ${ }^{33}$

Output of the enteric defense program reproduces the same stereotyped motor behavior in response to exposure to radiation, mucosal contact with noxious stimulants, or antigenic detection by the sensitized enteric immune system. ${ }^{34}$ Whether FGID symptoms sometimes reflect paradoxical output of the program is unresolved. The neural program incorporates connections between myenteric and submucous plexuses that coordinate mucosal secretion with propulsive motor behavior. The program is organized to stimulate copious secretion that flushes the mucosa and suspends the offensive material in solution in the segment ahead of the powerful propulsive contractions, which, in turn, empty the lumen. The overall benefit is rapid excretion of material recognized by the immune system as threatening.

Several kinds of immune/inflammatory cells including lymphocytes, macrophages, polymorphonuclear leucocytes, and mast cells are putative sources of paracrine signals to the ENS. Signaling between mast cells and the neural elements of the local microcircuits is the best understood. Antigen-evoked degranulation of mast cells releases a variety of paracrine messengers that may include serotonin, histamine, prostaglandins, leukotrienes, plateletactivating factor, and cytokines (fig 4). Among these, histamine is implicated as a significant messenger in communication between the enteric immune system and the ENS in animal models.

Applications of histamine, to simulate degranulation of mast cells in a guinea pig model, evokes rhythmic bursts of electrolyte/water secretion coordinated with contraction of the musculature. ${ }^{3}$ Histamine $\mathrm{H}_{2}$ receptors on enteric neurons initiate the cyclic behavior. Several days after sensitization to either a parasite or food antigen, re-exposure to the antigen evokes a pattern of cyclical behavior like that seen during histamine application. ${ }^{35} 36$ The combination of evidence suggests that recognition of sensitizing antigens by intestinal mast cells leads to release of histamine, which signals activation of a neuronal pattern generator from the library of programs stored in the local neural network.

\section{Brain-mast cell connection for functional gastrointestinal disorders}

Enteric mast cells seem to be involved in defense mechanisms apart from local antigen sensing and signaling to the ENS. An hypothesis that mast cells are relay nodes for transmission of selective information from the brain to the ENS is plausible and of sufficient significance to justify attention. Evidence from ultrastructural and light microscopic studies suggests that enteric mast cells are innervated by projections from the CNS. ${ }^{37-39}$ Functional evidence supporting the brain to mast cell connection is found in reports of Pavlovian conditioning of mast cell degranulation in the gastrointestinal tract. ${ }^{40}$ Release of mast cell protease into the systemic circulation is a marker for degranulation of enteric mucosal mast cells. This can be demonstrated as a conditioned response in laboratory animals to either light or auditory stimuli and in humans as a conditioned response to stress, ${ }^{41}$ indicative of a brain to enteric mast cell connection. Findings that stimulation of neurons in the brain stem by thyrotropin releasing hormone (TRH) evokes degranulation of mucosal mast cells in the rat small intestine are additional evidence for brain-mast cell interactions. ${ }^{42}$ In the upper gastrointestinal tract of the rat, intracerebroventricular injection of TRH evokes the same kinds of gastric inflammation and erosions as cold-restraint stress. In the large bowel, restraint stress exacerbates nociceptive responses and these effects are associated with increased release of histamine. ${ }^{43}$ Intracerebroventricular injection of corticotropin releasing factor (CRF) mimics the responses to stress. Intracerebroventricular injection of a CRF antagonist or pretreatment with mast cell stabilizing drugs suppresses stressinduced responses.

Mast cell degranulation may release mediators that sensitize silent nociceptors in the large intestine. In animal models, degranulation of intestinal mast cells results in a reduced threshold for pain responses to balloon distension $^{44}$ that was prevented by treatment with mast cell stabilizing drugs.

\section{Implications of the brain-mast cell connection for functional gastrointestinal disorders}

The brain to mast cell connection appears to be a mechanism that can link psycho-emotional status to irritable states of the digestive tract. The irritable state of the bowel (abdominal discomfort and diarrhea), known to result from 
degranulation of intestinal mast cells and release of signals to the ENS, is expected to occur irrespective of the mode of stimulation of the mast cells (fig 3). Degranulation and release of mediators evoked by neural input will have the same effect on motility and secretory behavior as degranulation triggered by antigen detection. This may explain the similarity of bowel symptoms between those associated with noxious insults in the lumen and those associated with stress in susceptible individuals.

The immunoneurophysiologic evidence leads to the inescapable conclusion that the moment-to-moment behavior of the gut, whether it be normal or pathologic, is determined by integrative functions of the ENS. Informational input processed by the enteric minibrain is derived from local sensory receptors, immune/inflammatory cells (mast cells), and the CNS. Mast cells utilize the capacity of the immune system for detection of new antigens and long term memory that permits recognition of the antigen if it ever reappears in the gut lumen. Should the antigen reappear, mast cells signal its presence to the enteric minibrain. The minibrain interprets the mast cell signal as a threat and calls up from its program library, secretory and propulsive motor behavior organized for quick and effective eradication of the threat. Operation of the program protects the integrity of the bowel, but at the expense of the side effects of abdominal distress and diarrhea. The same symptomatology is expected to result from activation of neural pathways that link psychologic states in the brain to the mast cells in the gut. The immunoneurophysiology in this respect is suggestive of mechanisms with susceptibility to malfunctions that could result in symptoms resembling FGID.

\section{Central neurophysiology in psychiatric disorders and functional gastrointestinal disorders}

Modern methods of brain imaging ${ }^{45}$ have made it possible to map regions of the brain involved in cognitive processing and to compare normal subjects and patients with psychiatric disorders. Changes - for example, have been found in the ventral prefrontal cortex in patients with unipolar and familial forms of depression when compared with normal subjects. Decreased vascular perfusion seen in image scans of localized regions of the prefrontal cortex normalizes after recovery from the depressed state. ${ }^{46}$ Putative relationships between psychiatric disorders and $\mathrm{FGIDs}^{47}$ underscore a need for comparison of psychiatric and FGID patients with normal subjects. Application of brain imaging in FGIDs has begun, but is at an early stage. ${ }^{48} 49$ In view of the fact that brain imaging has identified abnormalities associated with psychiatric disorders, there is a need to repeat the same studies in well defined groups of patients with FGIDs in order to start the process of understanding the relationships for brain dysfunction in the two groups of disorders.

\section{Directions for the future}

NEUROGASTROENTEROLOGY

Many lines of evidence implicate dysfunction in the nervous system as a significant factor underlying symptomatology in patient complaints and behavior that fit criteria for FGID. This justifies future attention to the development of the subspeciality of neurogastroenterology. Neurogastroenterology encompasses the investigative sciences dealing with functions, malfunctions, and malformations in the brain and spinal cord and the sympathetic, parasympathetic, and enteric divisions of the autonomic innervation of the digestive tract. Psychologic and psychiatric relations to FGID are significant components of the neurgastroenterologic domain. Acceptance of neurogastroenterology as the name for the subspeciality of gastroenterology where the bulk of future progress in understanding FGID is expected and will undoubtedly escalate in the future. This should signal its acceptance as a bona fide field of gastroenterologic research and clinical practice. ${ }^{5051}$

\section{CENTRAL NERVOUS SYSTEM}

The CNS is key to understanding conscious perception of real gastrointestinal pain, genesis of non-painful sensations, the emotional consequences of real pain perception, and psychologic origins of projection of discomfort to the bowel. Spinal pathways and gating mechanisms for nociceptive and non-nociceptive sensations of gastrointestinal origin are poorly explored areas amenable to investigation with potential for understanding disordered sensory aspects of FGID. Advances in understanding the basic neurophysiology of nausea and vomiting are expected to focus on origins in the CNS. Targeting of basic mechanisms of nausea and vomiting for pharmacotherapy with agents such as the non-peptide NK-1 receptor antagonists holds future promise. Nevertheless, future research should not ignore evidence that the peripheral nervous system, especially vagal afferents, is of equal importance in the basic physiology and pharmacology of nausea and vomiting.

New technologies for imaging or otherwise detecting activity in the functioning brain have strong potential for better understanding of how malfunctions of central processing are related to symptoms in patients with FGID. These approaches will be necessary for distinguishing peripheral sensitization of sensory detection from abnormalities of central processing as underlying neuropathology in the hypersensitivity to gut pain in patients with IBS. They offer promise for improved insight into abnormality of processing in the brain nuclei involved in cognitive perception of gut sensations, integration into emotional consciousness and psychogenic aspects of behavioral phenotype.

\section{ENTERIC NERVOUS SYSTEM}

Consideration that the ENS is an independent integrative nervous system with most of the neurophysiologic complexities found in the CNS suggests that FGID symptoms may origi- 
nate there as well. Lack of understanding of how subtle malfunctions may occur in the synaptic microcircuits of the ENS is the basis of "functional" as the descriptor for several forms of disordered gastrointestinal motility. This is reminiscent of neurologic disorders, such as Parkinsonian tremors, ballisms, and choreas, that were classified as functional prior to understanding of neurotransmission in microcircuits of somatic motor centers in the brain. Like somatic motor control centers in the brain a half century in the past, the ENS remains a virtual black box that must be opened scientifically in order to acquire real understanding of FGID.

Acquisition of new knowledge of the neurobiology of the enteric minibrain will require application of the same methodologies that unified functional concepts for the CNS. Electrophysiologic and synaptic behavior of individual enteric neurons, identification of neurotransmitters, how specific neuronal types are wired into synaptic circuits and the emergent properties of microcircuits in the programing of motor and secretory behavior are areas open to innovative investigation. Further investigation of enteric sensory physiology and the influence of inflammation and noxious insult holds promise for understanding why the digestive tract is sensitized to distension in patients afflicted with IBS, functional dyspepsia, or non-cardiac chest pain.

ENTERIC NEUROIMMUNE INTERACTIONS

Study of the interactions of the enteric immune system and the ENS is an area where progress can be expected in understanding FGID. Enteric mast cells may be a key cell type responsible for signaling the ENS to program behavior that results in FGID-like symptoms, and for initiating inflammatory cascades that generate chemical mediators (e.g., cytokines) currently known to have potent actions on enteric neurons. Evidence that activation of enteric mast cells can occur by central nervous signals as well as local insults requires further exploration to determine whether a brain-mast cell connection underlies gut reactions to psychogenic stress.

Cases where autoimmune attack is targeted to enteric neurons require future investigative scrutiny because current evidence suggests that FGID-like symptoms may signal the onset of the immunologic event that culminates in symptoms of chronic pseudo-obstruction. This appears to be true for explained forms of neuropathic autoimmunity (paraneoplastic syndrome and Chagas disease) and the idiopathic form. Future directions should include attention to development of tests for enteric neuropathic autoimmunity that can be applied in diagnostic workups during early indications of FGID.

1 Wood JD. Electrical and synaptic behavior of enteric neurons. In: Wood JD, ed. Handbook of physiology. The gastrointestinal system, motility and circulation. Bethesda, MD: American Physiological Society, 1989:465-517.

2 Wood JD. Physiology of the enteric nervous system. In: Johnson LR, Alpers DH, Christensen J, et al, eds. Physiology of the gastrointestinal tract. New York: Raven Press, 1994:423-82.
3 Cooke HJ, Wang YZ, Rogers R. Coordination of $\mathrm{Cl}^{-}$ secretion and contraction by a histamine $\mathrm{H}_{2}$-recepto agonist in guinea pig distal colon. Am f Physiol 1993;265: G973-8.

4 Wood JD. Histamine signals in enteric neuroimmune interactions. Ann N Y Acad Sci 1992;664:275-83.

5 Magni G. The use of antidepressants in the treatment of chronic pain. A review of the current evidence. Drugs 1991;42:730-48.

6 Cervero F, Janig W. Visceral nocireceptor: a new world order? Trends Neurosci 1992;15:374-8.

7 Furness JB, Kunze WAA, Bertrand PP, et al. Intrinsic primary afferent neurons of the intestine. Prog Neurobiol 998;54:1-18.

8 Whitehead WE, Holtkotter B, Enck P, et al. Tolerance for rectosigmoid distension in irritable bowel syndrome. Gastroenterology 1990;98:1187-92.

9 Lembo T, Munakata J, Mertz H, et al. Evidence for the hypersensitivity of lumbar splanchnic afferents in irritable bowel syndrome. Gastroenterology 1994;107:1686-96.

10 Richter JE, Barish CF, Castell DO. Abnormal sensory perception in patients with esophageal chest pain. Gastroenterology 1986;91:845-62.

11 Löning-Baucke V. Sensitivity of the sigmoid colon and rectum in children treated for chronic constipation. 7 Pediatr Gastroenterol Nutr 1984;3:454-9.

12 White J. Sensory innervation of the viscera. Res Publ Assoc 1943;23:373-90.

13 Troncon LEA, Thompson DG, Ahuwalia NK, et al. Relations between upper abdominal symptoms and gastric distension abnormalities in dysmotility like functional dyspepsia and after vagotomy. Gut 1995;37:17-22.

14 Lewis T. Pain. New York: Macmillan, 1942.

15 Cervo F. Afferent activity evoked by natural stimulation of the biliary system in the ferret. Pain 1982;13:137-51.

16 Maggi CA, Catalioto RM, Criscuoli M, et al. Tachykinin receptors and intestinal motility. Can $\mathcal{F}$ Physiol Pharmacol 1997;75:696-703.

17 Cooke HJ. Role of the "little brain" in the gut in water and electrolyte homeostasis. FASEB F 1989;3:127-38.

18 Burnstock G. Purinergic neurons. Pharmacol Rev 1972;34: 509-81.

19 Jin JG, Murthy KS, Grider JR, et al. Stoichiometry of neurally induced VIP release, NO formation, and relaxation in rabbit and rat gastric muscle. Am F Physiol 1996;34: G357-69.

20 Stanghellini V, Camilleri M, Malagelada JR. Chronic idiopathic intestinal pseudo-obstruction. Clinical and intestinal manometric findings. Gut 1987;23:824-8.

21 Camilleri M, Phillips S. Disorders of small intestinal motility. Gastroenterol Clin North Am 1989;18:405-24.

22 Palo J, Haltia M, Carpenter S, et al. Neurofilament subunitrelated proteins in neuronal intranuclear inclusion. $A n n$ Neurol 1984;15:316-21.

23 Monoz-Garcia D, Ludwin S. Adult-onset neuronal intranuclear hyaline inclusion disease. Neurology 1986;36: 785-90.

24 Krishnamurthy S, Shuffler MD. Pathology of neuromuscular disorders of the small intestine and colon. Gastroenterology 1997;93:610-39.

25 DeGiorgio R, Bassotti G, Stanghellini V, et al. Clinical, morpho-functional and immunological features of idiopathic myenteric ganglionitis [abstract]. Gastroenterology 1996;110:A655.

26 Smith V, Gregson N, Foggensteiner L. Acquired intestinal aganglionosis and circulating autoantibodies without neoplasia or other neural involvement. Gastroenterology 1997; 112:1366-71.

27 Andrews PLR, Davis CJ. The physiology of emesis induced by anti-cancer therapy. In: Reynolds DJ, Andrews PLR, Davis CJ, eds. Serotonin and the scientific basis of anti-emetic therapy. Oxford: Oxford Clinical Communications, 1995: $525-49$

28 Lucot JB. 5- $\mathrm{HT}_{1 \mathrm{~A}}$ receptor agonists as anti-emetics. In: Reynolds DJ, Andrews PLR, Davis CJ, eds. Serotonin and the scientific basis of anti-emetic therapy. Oxford: Oxford Clinical Communications, 1995:222-7.

29 Rudd JA, Naylor RJ. Opioid receptor involvement in emesis and anti-emesis. In: Reynolds DJ, Andrews PLR, Davis CJ, eds. Serotonin and the scientific basis of anti-emetic therapy. Oxford: Oxford Clinical Communications, 1995: 208-21.

30 Andrews PLR, Bhandari P. Resiniferatoxin, an ultrapotent capsaicin analogue, has anti-emetic properties in the ferret. Neuropharmacology 1993;32:799-806.

31 Watson JW, Gonsalves SF, Fossa AJ et al. The role of the NK1 receptor in emetic responses: the anti-emetic effects of CP-99,994 in the ferret and the dog. Br f Pharmacol 1995;115:84-94

32 Wood JD. Neuro-immunophysiolgy of colon function. Pharmacology 1993;47(suppl 1):7-13.

33 Phillips SF. Motility disorders of the colon. In: Yamada T, Alpers DH, Owyang C, et al, eds. Textbook of gastroenterology. Philadelphia: JB Lippincott, 1995:1856-75.

34 Sarna SK, Otterson MF, Cowles VE, et al. In vivo motor response to gut inflammation. In: Collins SM, Snape WJ, eds. Effects of immune cells and inflammation on smooth muscle and enteric nerves. Boca Raton: CRC Press, 1991:18195 .

35 Frieling T, Cooke HJ, Wood JD. Neuroimmune communication in the submucous plexus of guinea-pig colon after
sensitization to milk antigen. Am 7 Physiol 1994;267: G1087-93. 
36 Frieling T, Palmer JM, Cooke HJ, et al. Neuroimmune communication in the submucous plexus of guinea pig colon after infection with Trichinella spiralis. Gastroenterology 1994;107:1602-9.

37 Stead RH, Tomioka M, Quinonez G, et al. Intestinal mucosal mast cells in normal and nematode-infected rat intestines are in intimate contact with peptidergic nerves. Proc Natl Acad Sci USA 1987;84:2975-9.

38 Williams RM, Berthoud HR, Stead RH. Association between vagal afferent nerve fibers and mast cells in rat jejunal mucosa [abstract]. Gastroenterology 1995;108: A941.

39 Gottwald T, Lhotak S, Stead R. Effect of truncal vagotomy and capsaicin on mast cells and IgA-positive plasma cells in rat jejunal mucosa. Neurogastroenterol Motil 1997:9·2540.

40 MacQueen G, Marchall J, Perdue M, et al. Pavlovian conditioning of rat mucosal mast cells to secrete rat mast cell protease II. Science 1989;243:83-4.

41 Santos J, Saperas E, Nogueiras C, et al. Release of mast cell mediators into the jejunum by cold pain stress in humans. Gastroenterology 1998:114:640-8.

42 Santos J, Esteban S, Mourelle M, et al. Regulation of intestinal mast cells and luminal protein release by cerebral thyrotropin-releasing hormone in rats. Gastroenterology 1996;111:1465-73.
43 Gue M, Del Rio-Lacheze C, Eutamene H, et al. Stressinduced visceral hypersensitivity to rectal distension in rats: role of $271-9$.

44 Coelho AM, Fioramonti J, Bueno L. Mast cell degranulation induces delayed rectal allodynia in rats: role of histamine and 5-HT. Dig Dis Sci 1998;43:727-37.

45 Drevets W, Todd R. Neuroimaging in psychiatry. In: Guze S, ed. Adult psychiatry. St Louis: Mosby, 1997:53-82.

46 Bench C, Frackowiak R, Dolan R. Changes in regional blood flow on recovery from depression. Psychol Med 1995; 25:247-61.

47 North CS, Alpers DH, Thompson SJ, et al. Gastrointestinal symptoms and psychiatric disorders in the general population: Findings from the NIMH Epidemiologic Catchment Area Project. Dig Dis Sci 1996;41:633-40.

48 Aziz Q, Thompson D. Brain-gut axis in health and disease. Gastroenterology 1998;114:559-78.

49 Silverman DHS, Munakata JA, Ennes H, et al. Regional cerebral activity in normal and pathological perception of visceral pain. Gastroenterology 1997;112:64-72

50 Grundy D. The changing face of gastrointestinal motility. $\mathcal{F}$ Grundy D. The changing face of
Gastrointest Motil 1993;5:231-2.

51 Krammer H-J, Enck P, Tack J. Neurogastroenterology from the basics to the clinics. $Z$ Gastroenterol 1997;(suppl 2):1-68.

\title{
ROME II
}

\section{The Functional Gastrointestinal Disorders}

\author{
Publication date - January 2000
}

This book (over 500 pages) presents the full reports from 10 multinational working teams from which these articles have been summarized. Rome II not only details the criteria for system based diagnosis of functional gastrointestinal disorders but also presents the latest information on pathophysiology, diagnostic approach and treatment of 24 functional GI disorders.

The book includes an introductory overview chapter by Douglas Drossman on the functional gastrointestinal disorders and the rationale for the Rome process, a chapter by William Whitehead on the definition of responders in clinical trials resulting from a consensus conference in Vienna, and an historical chapter by Grant Thompson on the development of the multinational working teams over the past 10 years. A unique feature of this book is the inclusion of a questionnaire that can be used for surveys and clinical trials which contains all the Rome Criteria in questionnaire form.

This book will be used as both a textbook and an up-to-date reference source for anyone interested in clinical care of a comprehensive review of the field. It is must reading for primary care clinicians and researchers. In bringing this new medical knowledge from the multinational working teams to primary care physicians and gastroenterologists throughout the world, this book advances a symptom-based classification system developed as a new paradigm for the diagnosis and treatment of functional GI disorders so that patients suffering from these conditions may find the promise of relief contained in Rome II.

For additional information, visit our website at: www.romecriteria.org or e-mail inquiries to: giworkingteam@degnon.org 